\title{
Formability of the steel sheet at the intermediate strain rate
}

\author{
Hoon Huh ${ }^{1, a}$, Ji-Ho Lim ${ }^{1, b}$, Seok-Bong Kim,b, Soo-Sik Han ${ }^{2, d}$, Sung-Ho Park ${ }^{3, e}$ \\ ${ }^{1}$ Department of Mechanical Engineering, Korea Advanced Institute of Science and Technology, \\ Science town, Daejeon, 305-701, Korea \\ 2Department of Mechanical Engineering, Kumoh National Institute of Technology, 188, \\ Shinpyung-dong, Gumi, Gyungbuk 730-701, Korea \\ ${ }^{3}$ Automotive Steels Research Center, PSOCO, 699, Gumho-dong, Gwangyang-si, Jeonnam, \\ 545-090, Korea

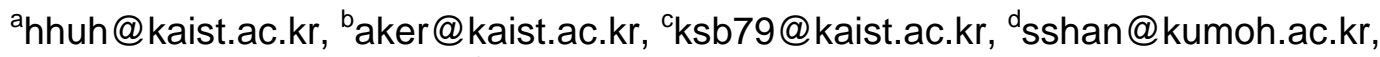 \\ esunghopark@posco.co.kr
}

Keywords: Elongation, Formability, Intermediate strain rate, Strain rate hardening.

\begin{abstract}
While the formability is important and indispensable for success in very complicated sheet metal forming, it seems that few studies has been carried out about the formability of sheet metal at the high strain rate. The present experimental results report that the elongation is dependent on the crosshead speed in tensile tests. In this paper, the tensile elongation has been obtained from various steel sheets for an auto-body at the intermediate strain rate. The strain rate in the experiment is ranged from $0.003 / \mathrm{sec}$ to $200 / \mathrm{sec}$. The experimental result demonstrates that the tensile elongation does not decrease as the strain rate increases. This tendency has varieties depending on the microstructure and forming history of sheet metal. Some high strength steels have the tendency that the tensile elongation increases as the strain rate increases, while others not. This phenomenon is very important not only in sheet metal forming but also in the crashworthiness evaluation to predict the fracture and tearing of sheet metal members.
\end{abstract}

\section{Introduction}

The sheet metal forming process is one of the most important fabrication processes for an auto-body and has enjoyed its prosperity with a burst of development of forming techniques. Owing to remarkable development of forming techniques, its capabilities have been extended to very complicated sheet metal forming and multi-stage forming. Although the formability is important and indispensable for success in very complicated sheet metal forming, few studies have been done about the formability of sheet metal since it was difficult to make the tensile test of sheet metal at the intermediate strain rate ranged from $1 / \mathrm{s}$ to $1000 / \mathrm{s}$.

Mechanical, pneumatic and servo-hydraulic loading methods have been used to measure the material property at the intermediate strain rate [1,2]. Dudder [3] and Ambur et al. [4] studied to obtain the material properties using a drop weight method and other researchers used a cam plastometer and a rotary flywheel machine and so on. Nowadays, servo-hydraulic material testing machines are employed in most research works [5, 6]. However, a few in-depth studies have been published about the dynamic material properties such as the yield and tensile strength as well as the tensile elongation at the intermediate strain rate.

In this paper, the tensile elongation has been obtained from various steel sheets for an auto-body at the intermediate strain rate. A high speed material testing machine was made for tension tests at the intermediate strain rate and the dimensions of specimens that can provide the reasonable results were determined by the finite element analysis. Dynamic tensile tests of seventeen different steel sheets for 
an auto-body were performed to investigate the relation between the strain rate and the tensile elongation. Stress-strain curves were obtained for each steel sheet from the dynamic tensile test and used to deduce the relationship of the yield stress and the elongation to the strain rate.

\section{Dynamic material tests at the intermediate strain rate}

\subsection{High speed material testing machine}

A high speed material testing machine of the servo-hydraulic type was made in our laboratory as shown in Fig. 1 in order to obtain the dynamic material properties at the intermediate strain rate. The maximum velocity and load of the apparatus are $4000 \mathrm{~mm} / \mathrm{s}$ and $30 \mathrm{kN}$ respectively. Two electric motors are used to compress the operating oil up to the pressure of 300 bars and two accumulators with the capacity of 5 liters are used to make the response time faster. The maximum flow rate of the servo-hydraulic unit is $240 \mathrm{liter} / \mathrm{min}$. The load is acquired by a piezoelectric-type load cell, Kistler 9051A, and the displacement is obtained by a LDT (linear displacement transducer) from Sentech company.

While the moving gripper needs to maintain the constant velocity during tensile tests, the moving gripper can not operate at the desired velocity in an instant even if a servo valve with the fastest response time is used. In order to achieve the constant velocity during tests, a special jig is used to move to some distance under no loading and then seize a specimen instantly.

As the strain rate increases, the transmitted load does not become uniformly distributed at a specimen, a jig and a load cell with oscillation since the inertia and stress wave affect parts of the equipment. This phenomenon is called the load ringing. The load ringing distorts experimental results and brings about the inaccurate or false conclusion. The load ringing can be reduced by increasing the natural frequency of an upper jig or by acquiring the load from a specimen directly. The load ringing has been remarkably diminished by designing a new simple jig for the upper gripper and load cell in the high speed material testing machine.

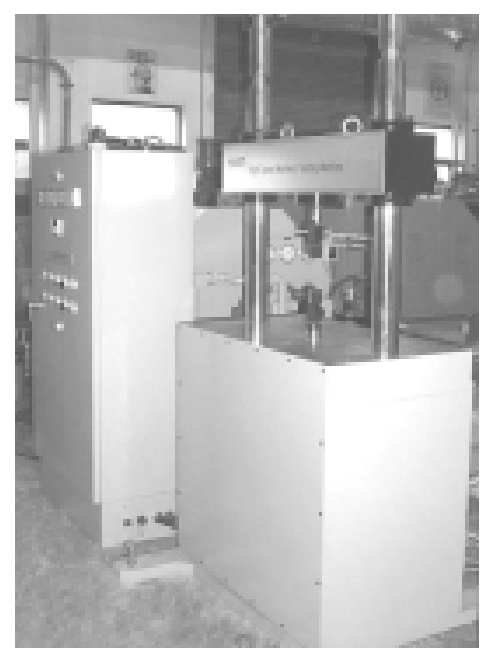

(a)

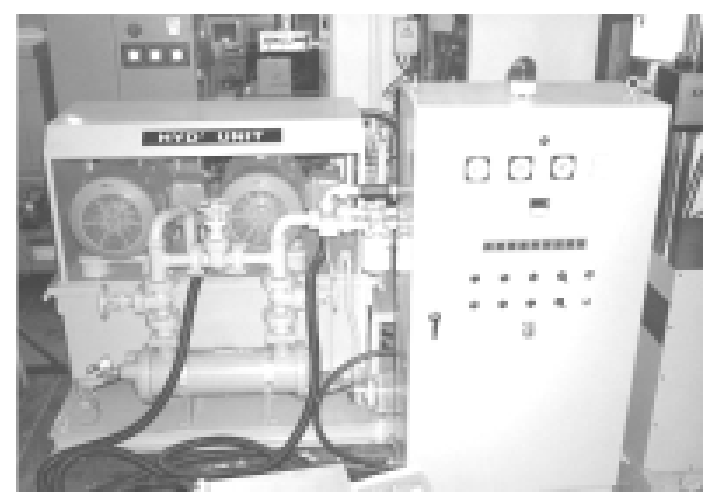

(b)

Fig. 1 High speed tension testing machine: (a) loading frame; (b) servo-hydraulic unit.

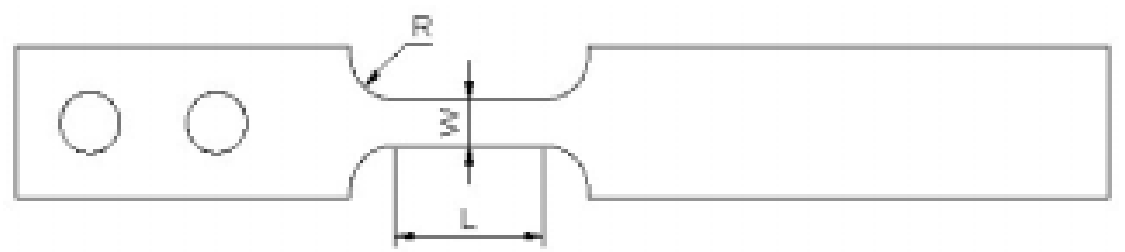

Fig. 2 Schematic diagram of a specimen. 


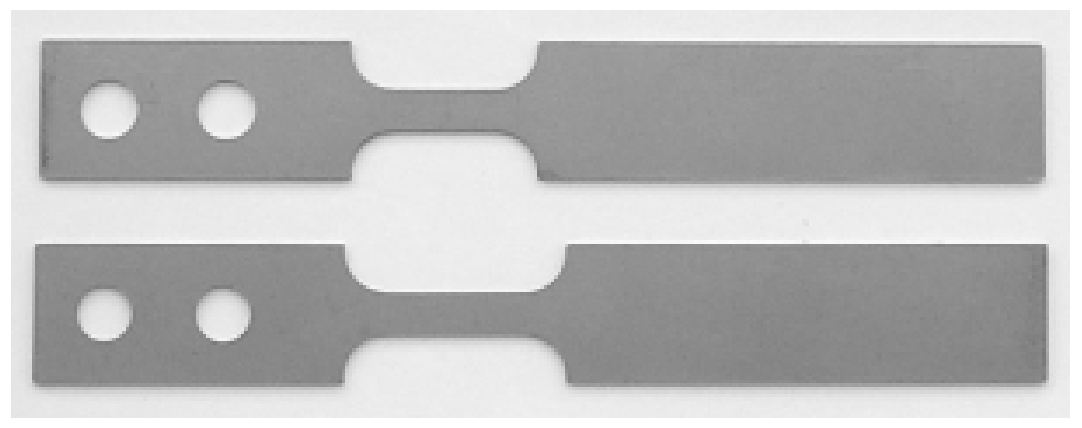

Fig. 3 Tensile specimens with the length of the gauge section of $15 \mathrm{~mm}$ and $20 \mathrm{~mm}$.

\subsection{Specimens in the intermediate strain rate test}

Specimens for the tensile test of a sheet metal at the intermediate strain rate is not yet defined in any material handbook. A tensile specimen of a sheet metal generally has the shape like that in Fig. 2. Major dimensions of a specimen are the length (L), the width (W) of the gauge section and the radius of the fillet $(\mathrm{R})$. It is important to determine the dimensions of a tensile specimen since the strain calculated from the displacement of the crosshead is different from the exact strain at the gauge section due to the deformation at the filleting section.

Good specimens have characteristics that most deformation occurs in the gauge section with uniform distribution. As the length of the gauge section increases, the error of the strain calculated from the displacement of the crosshead decreases, but the deformation tend to concentrate locally near the loading region due to the inertia effect. The width of a specimen needs to be within an allowable limit since the large width of a specimen can induce large deviation of the deformation between the side and inner region. The radius of the fillet has influence on stress concentration at both ends of the gauge section. The finite element analysis was used to determine dimensions of a specimen. The error of the strain calculated from the displacement of the crosshead is investigated when the width of the gauge section and the radius of fillet are fixed by $6 \mathrm{~mm}$ and the length of the gauge section is varied from $10 \mathrm{~mm}$ to $35 \mathrm{~mm}$. Specimens are selected with the length of the gauge section of $15 \mathrm{~mm}$ and $20 \mathrm{~mm}$ of which the error is acceptable as shown in Fig. 3.

\section{Investigation of the elongation for steel sheets according to strain rate}

The material properties at the intermediate strain rate were not acquired directly from the experiment because of difficulties of the experiment, but were obtained from interpolation of quasi-static test results and high strain rate test results from a split Hopkinson bar [7]. Development of a servo-hydraulic testing machine enables to obtain accurate material properties at the intermediate

Table 1 Experimental conditions.

\begin{tabular}{|c|c|c|c|}
\hline Machine & Strain Rate [/s] & $\begin{array}{c}\text { Length of gauge } \\
\text { section [mm] }\end{array}$ & Quantity \\
\hline Static UTM & 0.003 & 20 & 3 \\
\hline \multirow{4}{*}{$\begin{array}{c}\text { Material Testing } \\
\text { Machines }\end{array}$} & 0.1 & 20 & 3 \\
\cline { 2 - 4 } & 0.5 & 20 & 3 \\
\cline { 2 - 4 } & 1 & 20 & 3 \\
\cline { 2 - 4 } & 2 & 20 & 3 \\
\cline { 2 - 4 } & 5 & 20 & 3 \\
\cline { 2 - 4 } & 10 & 20 & 3 \\
\cline { 2 - 4 } & 20 & 20 & 3 \\
\cline { 2 - 4 } & 20 & 20 & 3 \\
\hline
\end{tabular}




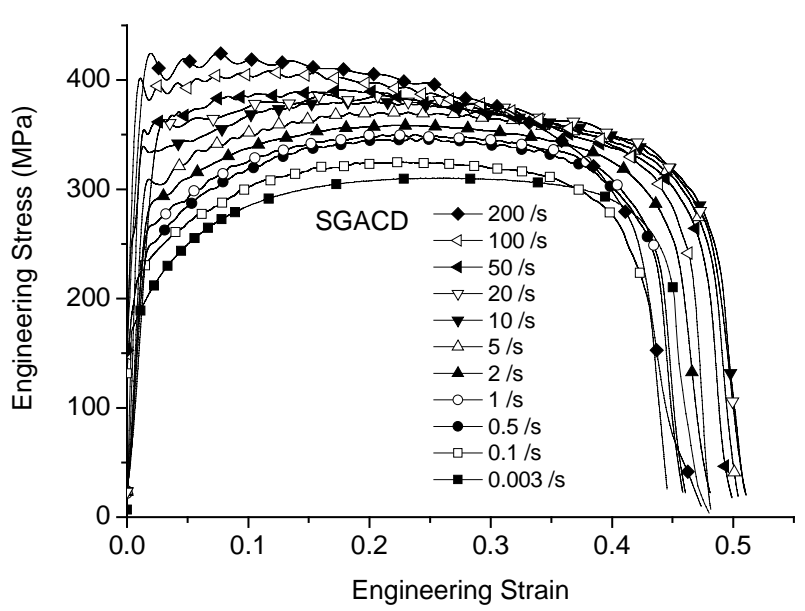

(a)

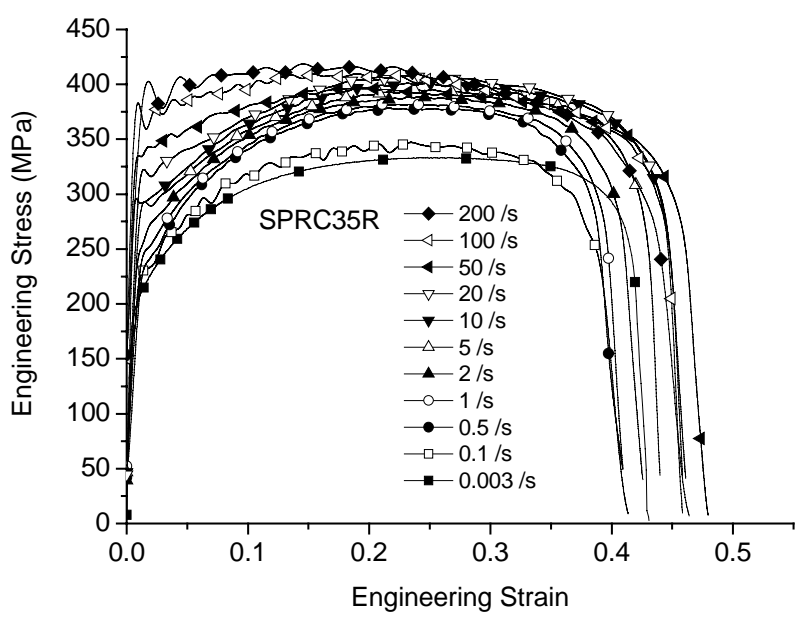

(b)

Fig. 4 Stress-strain curves at various strain rate: (a) SGACD; (b) SPRC35R.

strain rate. Table 1 shows experimental conditions of tensile tests that were carried out for several steel sheets for an auto-body at the intermediate strain rate. Seventeen materials were tested and typical results are presented for explanation: SPCC; SGACD; SPRC35R; and SPRC40R. The strain rate was varied from $0.003 / \mathrm{s}$ to $200 / \mathrm{s}$. The specimen with the length of the gauge section of $20 \mathrm{~mm}$ is used in tensile tests at the strain rates from $0.003 / \mathrm{s}$ to $100 / \mathrm{s}$ and the specimen with the length of the gauge section of $15 \mathrm{~mm}$ is adopted at the strain rate of $200 / \mathrm{s}$. Tests were repeated three times per each strain rate.

Stress-strain curves of SGACD and SPRC35R are shown in Fig. 4 at various strain rates. As the strain rate increases, the tensile strength gradually increases but the slope of the stress-strain curve decreases. The amount of decrease in the tensile strength varies with materials. As a result, the ultimate tensile strength occurs at the lower strain and the hardening exponent becomes smaller with the increase of the strain rate. This phenomenon seems to occur with the mechanism of grain sliding and the heat dissipated from the plastic work at the high strain rate.

The variation of the yield stress is demonstrated in Fig. 5 with respect to the increase of the strain rate. As the strain rate increases, the yield stress remarkably increases. The yield stress of low strength steel such as SGACD increases by more than two times while the increase of the yield stress of the high strength steel SPRC40R is about 1.5 times. In general, the yield stress of the low strength steel is more sensitive to the strain rate than that of the high strength steel.

The tensile elongation is depicted in Fig. 6 with respect to the log scale of the strain rate. In contrast to the conventional concept that the tensile elongation is generally decreased as the strain rate is increased, experimental results of steel sheets reveal a different viewpoint. The elongation is inversely proportional to the strain rate at the low strain rate up to $0.5 / \mathrm{s}$. However the elongation increases at the range from $0.5 / \mathrm{s}$ to about 10 20/s and decreases again above 10 20/s as the strain rate increases. It is noted from the experiment that as the strain rate increases, the development of necking is restrained and the necking spreads to the neighboring region. The strain rate at the necking region becomes particularly higher than the other region and due to the rapid increase of the strain rate the flow stress in the necking region exceeds the flow stress in the other region. Consequently, the necking region becomes stronger than the neighboring region despite of the thinning in the necking region. This phenomenon is closely related to the rate of increase in the yield stress. The increase rate of the yield stress is rather small at the strain rate ranged form $10^{-3}$ to $0.5 / \mathrm{s}$ while that becomes larger at the strain rate of above $0.5 \sim 1 / \mathrm{s}$.

This phenomenon is very interesting results different from the previous conjecture. The fact is very important not only in the formability assessment of the sheet metal forming but also in the crashworthiness evaluation to predict the fracture and tearing of steel sheet members of an auto-body. 


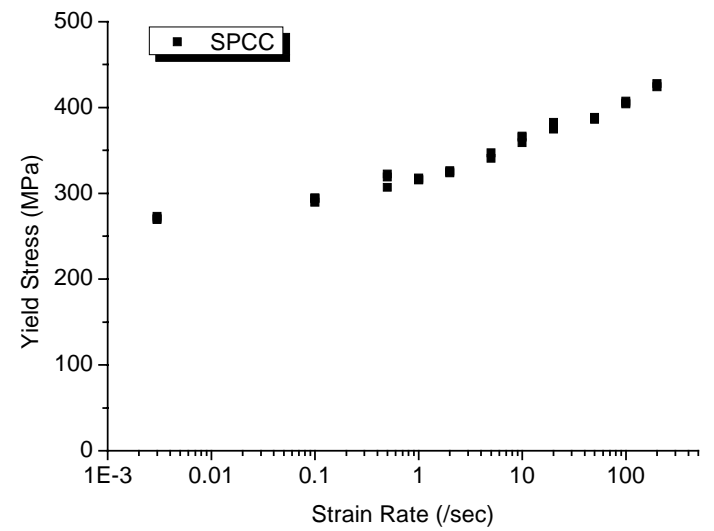

(a)

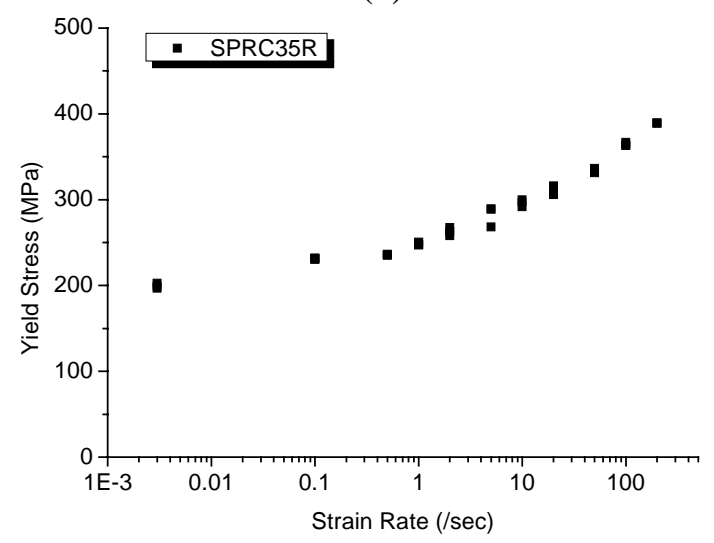

(c)

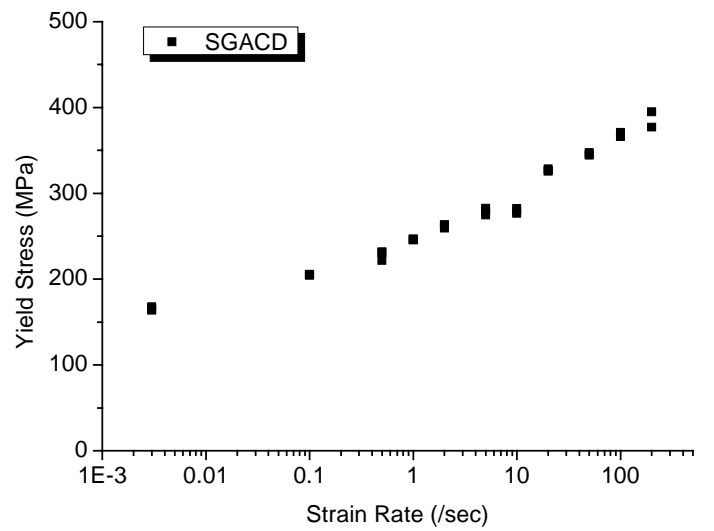

(b)

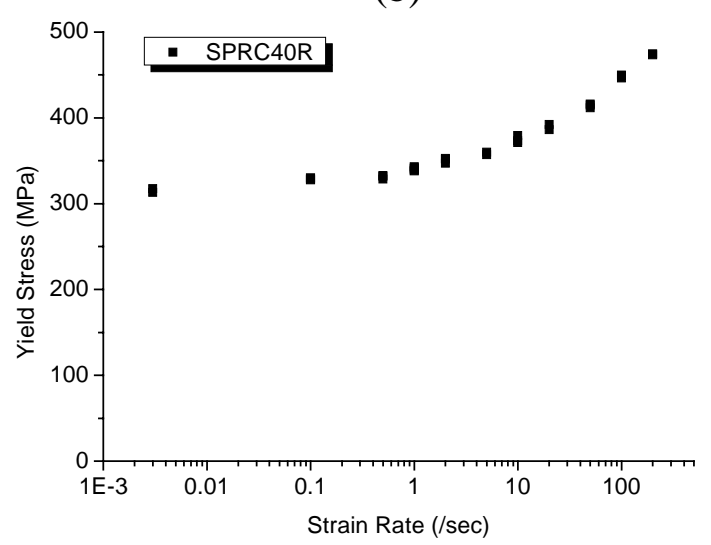

(d)

Fig. 5 Yield stress according to strain rate: (a) SPCC; (b) SGACD; (c) SPRC35R; (d) SPRC40R.

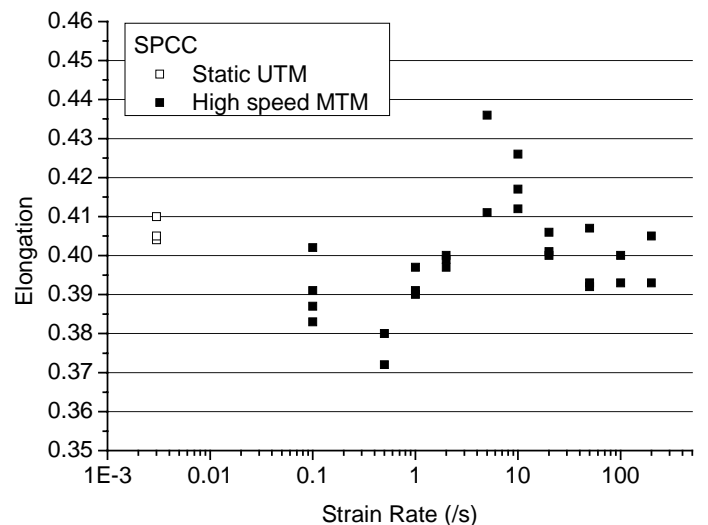

(a)

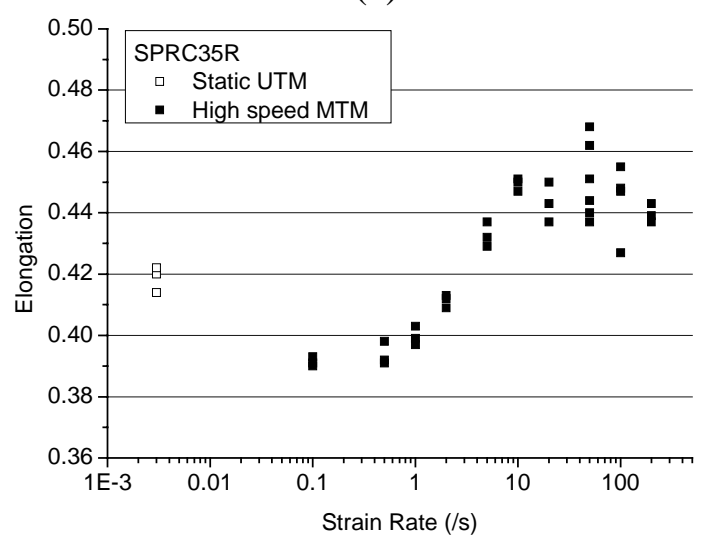

(c)

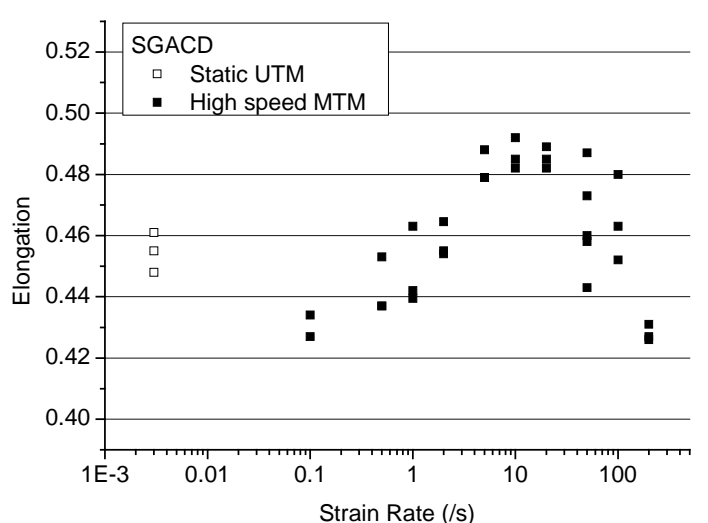

(b)

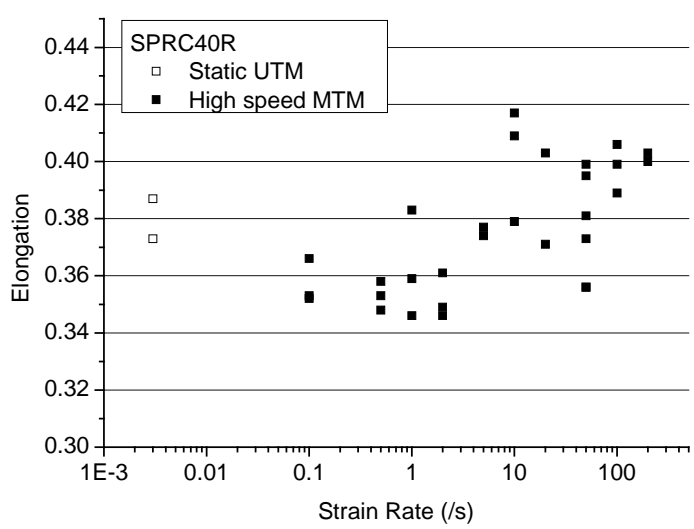

(d)

Fig. 6 Elongation according to strain rate: (a) SPCC; (b) SGACD; (c) SPRC35R; (d) SPRC40R. 
It means that fast forming rate can enhance the formability for secure forming process and the car crash at the moderate speed do not result in fracture or tearing of steel sheet at smaller strain than the quasi-static state.

\section{Conclusion}

This paper is concerned with the tensile elongation that has been obtained for various steel sheets for an auto-body at the intermediate strain rate. A high speed material testing machine was made for tensile tests at the intermediate strain rate and specimens that can provide the acceptable result were determined by the finite element analysis. Dynamic tensile tests of seventeen different steel sheets for an auto-body were performed to investigate the relation between the strain rate and the elongation. The relations of the yield stress and the elongation to the strain rate were obtained from the stress-strain curves at various strain rates.

In contrast to the conventional concept that the tensile elongation is generally decreased as the strain rate is increased, experimental results of steel sheets reveal a different viewpoint. The elongation is decreased as the strain rate is increased at the low strain rate up to $0.5 / \mathrm{s}$. However the elongation increases at the range from 0.5 /s to about 10 20/s and decreases again above 10 20/s as the strain rate increases. This phenomenon is very interesting and important in sheet metal forming and the high speed deformation such as the car crash from a viewpoint that the increment of the strain rate does not deteriorate the elongation.

\section{Reference}

[1] M. A. Meyers: Dynamic Behavior of materials, John Wiley \& Sons, New York (1994).

[2] Mechanical Testing, ASM Handbook, ASM Intl., Metals Park, Ohio, Vol. 8 (1985).

[3] G.B. Dudder: Drop Tower Compression Test, Metals Handbook, 9th Ed., American Society for Metals (1985), pp. 196-199.

[4] D.R. Ambur, C.B. Prasad and W.A. Waters Jr.: Experimental Mechanics, Vol. 3 (1995), pp.77-82.

[5] K. Miura, S. Takagi, T. Obara, and S. Tanimura: SAE, 980952 (1998).

[6] Instron Corporation, Operating Instructions of Instron FastTrack 8800 and Materials Test Control Systems (2002).

[7] H. Huh, W. J. Kang, and S. S. Han: Experimental Mechanics, Vol. 42 (2002), pp. 8-17. 